\section{Targeted PET imaging for prostate-specific membrane antigen in prostate cancer}

\author{
“As we progress to more and more accurate imaging modalities for \\ prostate cancer, we are able to visualize and localize disease that was \\ previously missed. Imaging using prostate-specific membrane antigen \\ targeted PET is currently the most accurate imaging option available, \\ but we likely are not seeing the entire distribution of metastatic disease \\ in these patients.”
}

\section{Thomas A Hope ${ }^{*, 1,2}$, Rahul R Aggarwal ${ }^{3}$, Antonio C Westphalen', Matthew R Cooperberg ${ }^{4}$ \& Kirsten L Greene ${ }^{4}$}

First draft submitted: 1 July 2016; Accepted for publication: 12 July 2016; Published online: 25 July 2016

\section{Prostate-specific membrane antigen imaging agents \\ The prostate-specific membrane antigen (PSMA) is a transmembrane protein over- expressed in the large majority of prostate cancers [1]. Several PSMA targeted radi- opharmaceuticals have been developed since 2008, the most well studied being gallium-68 $\left.{ }^{68} \mathrm{Ga}\right)-P S M A-11[2]$. In addi- tion to ${ }^{68} \mathrm{Ga}$-PSMA-11, other radionu- clides, for example, indium and fluori- nated agents, can be ligated to the same urea moiety of the PSMA protein and used for imaging [3]. Two studies that compared ${ }^{68} \mathrm{Ga}$-PSMA-11 to fluorocholine showed ${ }^{68} \mathrm{Ga}$-PSMA-11 localized more sites of disease, in particular in patients with a prostate-specific antigen (PSA) less than $2.0 \mathrm{ng} / \mathrm{ml}[4,5]$. PSMA PET, specifically}

utilizing ${ }^{68} \mathrm{Ga}-\mathrm{PSMA}-11$, has become available at a large number of institutions in the last 2 years, and may ultimately provide the community with a highly accurate imaging agent for prostate cancer across the spectrum of the disease.

\section{Preprostatectomy patients}

Prostatectomy is increasingly being used to treat high-risk prostate cancer patients [6], and as a consequence there is an urgent need for more accurate tools to detect regional and distant metastases prior to surgery. Recent studies looking at the diagnostic accuracy of ${ }^{68} \mathrm{Ga}-\mathrm{PSMA}-11$ PET for the detection of lymph node metastases in intermediate to high-risk prostate cancer patients prior to prostatectomy showed a per patient sensitivity of approximately of $64-66 \%[7,8]$.

\section{KEYWORDS}

- PET • prostate cancer

- prostate-specific membrane antigen • PSMA

\section{“The prostate-specific membrane antigen is a transmembrane protein overexpressed in the large majority of prostate cancers."}

'Department of Radiology \& Biomedical Imaging, University of California, San Francisco, San Francisco, CA, USA 2Department of Radiology, Veterans Affairs Medical Center, University of California, San Francisco, San Francisco, CA, USA

${ }^{3}$ Division of Hematology/Oncology, Department of Medicine, University of California, San Francisco, San Francisco, CA, USA 


\section{"As clinicians migrate toward more extirpative treatments, we as an imaging community need to demonstrate the clinical value of these approaches, and practice guidelines need to be updated to include imaging modalities that have increased detection sensitivity."}

Management of patients with positive imaging findings remains controversial, as it is unknown whether a positive finding on ${ }^{68} \mathrm{Ga}-\mathrm{PSMA}-11$ PET is a marker for widespread disease and need of systemic therapy, or if it represents local disease that can be treated with extended nodal dissection. To date, there have been no follow-up studies to offer an insight into the appropriate management of these patients.

Little data exist investigating the role of PSMA PET in the assessment of low-risk prostate cancer patients, but it is speculated that it could be used to detect clinically significant tumor, improving upon conventional multiparametric MRI. Until well-designed studies are completed and we have a better understanding of the role of PSMA PET in this population, it will be mostly reserved for the assessment of metastatic disease.

\section{Biochemical recurrence patients}

Patients with biochemical recurrence all have disease, which may be local, regional, and/or metastatic. The role of imaging in this scenario is to localize disease to determine the appropriate management, systemic therapy versus targeted treatment. Small case series have shown that targeted radiotherapy or pelvic nodal dissection of oligometastatic PSMA, positive lesions can result in prolonged biochemical control $[9,10]$. In the largest study to date, Eiber et al. estimated that PSMA PET detects recurrent cancer in up $97 \%$ for patients with a PSA $>2.0 \mathrm{ng} / \mathrm{ml}$, but only in $58 \%$ when the PSA is $<0.5 \mathrm{ng} / \mathrm{ml}[11]$, numbers superior to choline PET and other conventional imaging techniques [12].

\section{Treatment response \& osseous metastases}

Detection of osseous metastases and the assessment of their treatment remains a major limitation in imaging of prostate cancer patients. The detection of metastases with conventional bone scan with technetium-99m methylene diphosphonate or 18-sodium fluoride rely on the development of a sclerotic reaction to the metastasis, and lesions that are located in the bone marrow or that are predominantly lytic can be missed. A flare response can also limit the ability of conventional imaging to diagnose treatment response $[13,14]$. There are little data in the literature describing the use of PSMA PET for evaluation of treatment response, but it remains an appealing choice given the alternatives. One possible confounding factor is that effective targeting of the androgen receptor by treatment can result in an increase in PSMA expression, which to date has only been demonstrated in cell lines and animal models [15]. This PSMA 'flare' response may mask true treatment response early in the course of androgen receptor-targeted treatments. Future work focusing on the role of PSMA PET for the detection and characterization of osseous metastases is warranted.

\section{Castrate-resistant patients}

In castrate-resistant prostate cancer patients, the question is not related to detection of disease. Typically there are visualized sites of disease at this stage, but the question is whether PSMA PET can be used to characterize disease or predict response to therapies. For example, patients with osseous only metastatic disease are often treated with radium $(\mathrm{Ra})-223$, but it is unclear if the identification of soft tissue metastases solely on PSMA PET should preclude its use for treatment of such patients. Also, the recent development of PSMA targeted radiotherapy has opened up a new avenue for treatment. Peptide receptor radionuclide therapy uses compounds that are similar to those used for imaging, but instead of being positron emitters (e.g., ${ }^{68} \mathrm{Ga}$ or ${ }^{18} \mathrm{~F}$ ), they are with $\beta$ emitters (e.g., ${ }^{90} \mathrm{Y}$ or ${ }^{177} \mathrm{Lu}$ ) that accumulate in and kill cells. These small molecules have faster blood clearance than the large antibodies previously studied, and therefore have less toxicity associated with treatments. This application has shown promise in early studies treating castrate-resistant prostate cancer patients [16].

\section{Need for clinical trials in the future}

The majority of papers to date focus on detection of lesions in patients, using percent of patients with disease or correlation to pathology as their end points. What is missing from the literature are trials that demonstrate that localizing disease effects the outcome of patients by leading to effective targeted treatment. Currently, treating physicians assume that disease is limited to sites visualized on ${ }^{68} \mathrm{Ga}$-PSMA-11 PET studies and frequently are changing from systemic therapy to local therapies such as surgical lymphadenectomies or external beam radiation therapy. Anecdotally, patients frequently recur soon after treatment outside of the treated field. Therapeutic prostate cancer trials use overall mortality and other lengthy end points, but these may be difficult targets to reach for PSMA 
trials. Such end points take a long time to occur and are not necessarily frequent, making such trials expensive and time consuming. We must be creative and find alternative surrogates that reflect these long-term outcomes. One could consider, for example, to focus on the time to biochemical recurrence or the length of androgen deprivation therapy break. The results of future trials are imperative to the development of revised risk stratification tools and treatment guidelines.

\section{Conclusion}

As we progress to more and more accurate imaging modalities for prostate cancer, we are able to visualize and localize disease that was previously missed. Imaging using PSMA targeted PET is currently the most accurate imaging option available, but we likely are not seeing the entire distribution of metastatic disease in these patients. How do we determine in which patients with oligometastatic disease will targeted treatments be curative compared with those where systemic therapies should be the first-line therapy? Is PSMA PET simply showing the tip of the iceberg? As clinicians migrate toward more extirpative treatments, we as an imaging community need to demonstrate the clinical value of these approaches, and practice guidelines need to be updated to include imaging modalities that have increased detection sensitivity.

\section{Financial \& competing interests disclosure}

TA Hope receives grant support and is on the speaker's bureau for GE Healthcare. The authors have no other relevant affliations or financial involvement with any organization or entity with a financial interest in or financial conflict with the subject matter or materials discussed in the manuscript apart from those disclosed.

No writing assistance was utilized in the production of this manuscript.

\section{References}

Papers of special note have been highlighted as: - of interest; $\bullet$ of considerable interest

1 Israeli RS, Powell CT, Corr JG, Fair WR, Heston WD. Expression of the prostatespecific membrane antigen. Cancer Res. 54(7), 1807-1811 (1994).

2 Afshar-Oromieh A, Malcher A, Eder M et al. PET imaging with a $\left[{ }^{68} \mathrm{Ga}\right]$ gallium-labelled PSMA ligand for the diagnosis of prostate cancer: biodistribution in humans and first evaluation of tumour lesions. Eur. J. Nucl. Med. Mol. Imaging 40 (4), 486-495 (2012).

- First human experience using ${ }^{68}$ Ga-PSMA-11.

3 Rowe SP, Gorin MA, Allaf ME et al. PET imaging of prostate-specific membrane antigen in prostate cancer: current state of the art and future challenges. Prostate Cancer Prostatic Dis. doi:10.1038/pcan.2016.13 (2016) (Epub ahead of print).

4 Morigi JJ, Stricker PD, van Leeuwen PJ et al. Prospective comparison of ${ }^{18} \mathrm{~F}$-fluoromethylcholine versus ${ }^{68} \mathrm{Ga}$-PSMA $\mathrm{PET} / \mathrm{CT}$ in prostate cancer patients who have rising PSA after curative treatment and are being considered for targeted therapy. J. Nucl. Med. 56(8), 1185-1190 (2015).

-• Intrapatient comparison of ${ }^{68} \mathrm{Ga}-\mathrm{PSMA}-11$ and fluorocholine with lower prostatespecific antigen levels demonstrating the increased performance characteristics of prostate-specific membrane antigen PET.
5 Afshar-Oromieh A, Zechmann CM, Malcher A et al. Comparison of PET imaging with a ${ }^{68} \mathrm{Ga}$-labelled PSMA ligand and ${ }^{18} \mathrm{~F}$-cholinebased PET/CT for the diagnosis of recurrent prostate cancer. Eur. J. Nucl. Med. Mol. Imaging 41(1), 11-20 (2014).

6 Cooperberg MR, Carroll PR. Trends in management for patients with localized prostate cancer, 1990-2013. JAMA 314(1), 80-82 (2015).

7 Maurer T, Gschwend JE, Rauscher I et al. Diagnostic efficacy of ${ }^{68}$ Gallium-PSMA positron emission tomography compared with conventional imaging for lymph node staging of 130 consecutive patients with intermediate to high risk prostate cancer. J. Urol. 195(5), 1436-1443 (2016).

-• Largest preprostatectomy case series with pathology correlates in 130 patients.

8 van Leeuwen PJ, Emmett L, Ho B et al. Prospective evaluation of ${ }^{68} \mathrm{Gallium}$-PSMA positron emission tomography/computerized tomography for preoperative lymph node staging in prostate cancer. BJU Int. doi:10.1111/bju.13540 (2016) (Epub ahead of print).

9 Henkenberens C, Klot von CA, Ross TL et al. ${ }^{68} \mathrm{Ga}$-PSMA ligand PET/CT-based radiotherapy in locally recurrent and recurrent oligometastatic prostate cancer: early efficacy after primary therapy. Strablenther. Onkol. 192(7), 431-439 (2016).
10 Hijazi S, Meller B, Leitsmann C et al. Pelvic lymph node dissection for nodal oligometastatic prostate cancer detected by ${ }^{68} \mathrm{Ga}$-PSMA-positron emission tomography/ computerized tomography. Prostate 75(16), 1934-1940 (2015).

- First attempt to look at outcomes from curative treatment (nodal dissection) in patients with oligometastatic disease as defined by prostate-specific membrane antigen PET.

11 Eiber M, Maurer T, Souvatzoglou M et al. Evaluation of hybrid ${ }^{68} \mathrm{Ga}$-PSMA ligand PET/ CT in 248 patients with biochemical recurrence after radical prostatectomy. J. Nucl. Med. 56(5), 668-674 (2015).

-• Largest published series of biochemical recurrence patients imaged using ${ }^{68}$ Ga-PSMA-11.

12 Evangelista L, Briganti A, Fanti S et al. New clinical indications for ${ }^{18} \mathrm{~F} /{ }^{11} \mathrm{C}$-choline, new tracers for positron emission tomography and a promising hybrid device for prostate cancer staging: a systematic review of the literature. Eur. Urol. 70(1), 161-175 (2016).

13 Ryan CJ, Shah S, Efstathiou E et al. Phase II study of abiraterone acetate in chemotherapynaive metastatic castration-resistant prostate cancer displaying bone flare discordant with serologic response. Clin. Cancer Res. 17(14), 4854-4861 (2011).

14 Messiou C, Cook G, Reid AHM et al. The $\mathrm{CT}$ flare response of metastatic bone disease 
EDITORIAL Hope, Aggarwal, Westphalen, Cooperberg \& Greene

in prostate cancer. Acta Radiol. 52(5), 557-561 (2011).

15 Evans MJ, Smith-Jones PM, Wongvipat J et al. Noninvasive measurement of androgen receptor signaling with a positron-emitting radiopharmaceutical that targets prostatespecific membrane antigen. Proc. Natl Acad. Sci. USA 108(23), 9578-9582 (2011).

16 Baum RP, Kulkarni HR, Schuchardt C et al. ${ }^{177} \mathrm{Lu}$-labeled prostate-specific membrane antigen radioligand therapy of metastatic castration-resistant prostate cancer: safety and efficacy. J. Nucl. Med. 57(7), 1006-1013 (2016). 